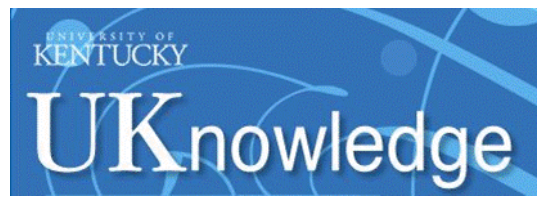

University of Kentucky

UKnowledge

$3-1-2020$

\title{
Contributions of Early Care and Education Programs to Diet Quality in Children Aged 3 to 4 Years in Central North Carolina
}

\author{
Courtney T. Luecking \\ University of Kentucky, courtney.luecking@uky.edu \\ Stephanie Mazzucca \\ Washington University in St Louis \\ Amber E. Vaughn \\ University of North Carolina at Chapel Hill \\ Dianne S. Ward \\ University of North Carolina at Chapel Hill
}

Follow this and additional works at: https://uknowledge.uky.edu/foodsci_facpub

Part of the Dietetics and Clinical Nutrition Commons

Right click to open a feedback form in a new tab to let us know how this document benefits you.

\section{Repository Citation}

Luecking, Courtney T.; Mazzucca, Stephanie; Vaughn, Amber E.; and Ward, Dianne S., "Contributions of Early Care and Education Programs to Diet Quality in Children Aged 3 to 4 Years in Central North Carolina" (2020). Dietetics and Human Nutrition Faculty Publications. 23.

https://uknowledge.uky.edu/foodsci_facpub/23

This Article is brought to you for free and open access by the Dietetics and Human Nutrition at UKnowledge. It has been accepted for inclusion in Dietetics and Human Nutrition Faculty Publications by an authorized administrator of UKnowledge. For more information, please contact UKnowledge@lsv.uky.edu. 
Contributions of Early Care and Education Programs to Diet Quality in Children Aged 3 to 4 Years in Central North Carolina

Digital Object Identifier (DOI)

https://doi.org/10.1016/j.jand.2019.09.018

Notes/Citation Information

Published in Journal of the Academy of Nutrition and Dietetics, v. 120, issue 3.

Copyright @ 2020 Academy of Nutrition and Dietetics

This manuscript version is made available under the CC-BY-NC-ND 4.0 license

https://creativecommons.org/licenses/by-nc-nd/4.0/.

The document available for download is the authors' post-peer-review final draft of the article. 
Title: Contributions of early care and education to 3- to 4-year-old children's diet quality in central North Carolina

Keywords or descriptive phrases: nutrition; diet quality; child care; family

Abstract word count: 303

Text word count: 3,443

\section{Author contact information:}

Courtney T Luecking, MPH, MS, RDN

Position: Doctoral Candidate, Department of Nutrition, Gillings School of Global Public Health, University of North Carolina at Chapel Hill

Address: 1700 Martin Luther King Jr. Blvd, CB 7426, Chapel Hill, NC 27599-7426

Telephone number: (618)-560-9924

Fax number: n/a

E-mail address: clueckin@email.unc.edu

Stephanie Mazzucca, PhD

Position: Assistant Professor, Prevention Research Center, Brown School, Washington

University in St. Louis

Address: One Brookings Drive, Campus Box 1196, St. Louis, MO 63130-4838

Telephone number: (314)-935-0117

Fax number: $\mathrm{n} / \mathrm{a}$

E-mail: smazzucca@wustl.edu 
Amber E Vaughn, MPH, RD

Position: Associate Director, Children's Healthy Weight Research Group, Center for Health

Promotion and Disease Prevention, University of North Carolina at Chapel Hill

Address: 1700 Martin Luther King Jr. Blvd, CB 7426, Chapel Hill, NC 27599-7426

Telephone number: (919)-843-0900

Fax number: n/a

E-mail: avaughn@email.unc.edu

Dianne S Ward, EdD, MS

Position: Professor, Department of Nutrition, Gillings School of Global Public Health and

Fellow, Center for Health Promotion and Disease Prevention, University of North Carolina at Chapel Hill

Address: 1700 Martin Luther King Jr. Blvd, CB 7426, Chapel Hill, NC 27599-7426

Telephone number: (919)-843-0901

Fax number: n/a

E-mail: $\underline{\text { dsward@email.unc.edu }}$

\section{Author Contributions:}

All authors contributed to conceptualization of this study. CTL supervised data management. CTL and SM performed data analysis. CTL and SM wrote the first draft. All authors reviewed and commented on subsequent drafts of the manuscript.

\section{Corresponding author:}


Dianne S Ward, EdD, MS

Children's Healthy Weight Research Group, Center for Health Promotion and Disease

Prevention, University of North Carolina at Chapel Hill, 1700 Martin Luther King Jr. Blvd, CB

7426, Chapel Hill, NC 27599-7426

Telephone number: (919)-843-0901

Fax number: n/a

E-mail: dsward@email.unc.edu

\section{Reprint contact:}

Dianne S Ward, EdD, MS

Contact information same as above

\section{Funding/financial disclosures:}

Funding for this project was provided by the National Heart, Lung, and Blood Institute of the National Institutes of Health (R01HL120969) The project was conducted out of the University of North Carolina at Chapel Hill at the Center for Health Promotion and Disease Prevention, a Prevention Research Center funded through a cooperative agreement with the Centers for Disease Control and Prevention (1U48DP005017). Support was also received from the Nutrition Obesity Research Center at the University of North Carolina at Chapel Hill (NIH DK056350) and the Washington University in St. Louis Center for Diabetes Translation Research (P30DK092950). Findings and conclusions of this article are those of the authors and do not necessarily represent the official position of the National Institutes of Health, National Heart, Lung, and Blood Institute, National Institute of Diabetes and Digestive and Kidney Diseases, or 
Centers for Disease Control and Prevention. The sponsor had no role in the study design; collection, analysis, or interpretation of the data; writing of the article; or decision to publish this article.

\section{Conflict of interest disclosures:}

Courtney Luecking, Stephanie Mazzucca, and Amber Vaughn declare that they have no conflicts of interest to report.

Declarations for potential conflicts of interest for Dianne Ward within the last five years are as follows: consulting for Healthy Eating Research, Robert Wood Johnson Foundation (RWJF) as a co-leader for the early care and education workgroup (2013-2017, $\$ 1,000$ per year); grant funding from Healthy Eating Research, RWJF to develop an online training to assess early care and education nutrition and physical activity environments (2014-2015, \$97,000); consulting for Nestle Nutrition Institute to prepare a paper on nutrition in early care and education settings (2018, \$3,000); consulting for Healthy Eating Research, RWJF to review grants (2018-2019; $\$ 550$ for 3 reviews), and; grant funding from the Kellogg Foundation for farm-to-ECE programming (2018-2019, \$180,600).

Acknowledgements: We would like to thank the child care centers and families who participated in the Healthy Me, Healthy We study as well as the project staff who supported participant recruitment, data collection, and quality assurance. 


\section{Research Snapshot}

2 Research question: What proportion of 3-4-year-old children's dietary intake occurs at early care

3 and education (ECE) centers? Is diet quality at ECE centers higher than away from ECE centers

4 and higher on weekdays compared to weekends?

5 Key findings: In this cross-sectional analysis of observed dietary intake of 840 3-4-year-old

6 children at ECE centers and the corresponding parent-reported dietary intake outside of child

7 care, children consumed approximately $40 \%$ of energy and nutrients at ECE centers. Diet

8 quality, as measured by the Healthy Eating Index-2015, was higher at ECE centers than away

9 from ECE centers and on weekdays than weekends (both $\mathrm{p}<0.0001$ ). 
10 Title: Contributions of early care and education to 3- to 4-year-old children's diet quality in

11 central North Carolina

12

13 Abstract:

14 Background. Parents and early care and education (ECE) are the key influencers of young

15 children's diets, but there is limited information about how each contribute to children's overall

16 diet quality.

17 Objective. This study aimed to determine what proportion of children's dietary intake occurs

18 within the ECE setting and whether diet quality is higher at ECE centers and, consequently, on

19 weekdays than weekends.

20 Design. This cross-sectional analysis of a larger cluster randomized controlled trial used multiple

21 24-hour dietary intakes measured through a combination of the Dietary Observation in Child

22 Care protocol and parent-reported food diaries.

23 Participants/setting. Participants $(\mathrm{n}=840)$ included children 3-4 years of age enrolled in ECE

24 centers in central North Carolina for whom 24-hour dietary intake was captured via observation

25 of meals and snacks consumed at ECE and parent-report of all remaining meals and snacks. Data

26 were collected from 2015 to 2016.

27 Main outcome measures. Diet quality at ECE and elsewhere was evaluated using the Healthy

28 Eating Index (HEI)-2015.

29 Statistical analyses performed. Mixed effects models were used to determine differences in mean

30 HEI-2015 component and total scores. Models were adjusted for children's age and sex and

31 accounted for clustering within ECE centers and families. 
32 Results. Children consumed approximately $40 \%$ of daily energy, nutrients, and food groups at

33 ECE centers. The mean total HEI-2015 score was higher for foods and beverages consumed at

34 ECE centers $(58.3 \pm 0.6)$ than elsewhere $(52.5 \pm 0.6)(\mathrm{p}<0.0001)$. The mean total HEI-2015 score

35 was also higher on weekdays $(58.5 \pm 0.5)$ than on weekends $(51.3 \pm 0.5)(\mathrm{p}<0.0001)$.

36 Conclusions. Children consume a majority of dietary intake away from ECE centers. Overall,

37 diet quality is low, but the quality of foods consumed by children at ECE centers is higher than

38 that consumed elsewhere. ECE centers remain an important source of nutrition and further

39 investigation is warranted to identify ways to support both ECE centers and families to provide

40 healthier eating environments. 


\section{Background}

42 Early childhood, defined as birth to 5 years of age, is a time of significant growth and

43 development that establishes the foundation for future physical, social, emotional, and cognitive

44 health. ${ }^{1,2}$ Dietary patterns and eating behaviors formed during this period have a lasting influence

45 on preferences, energy balance, and health in later childhood and into adulthood. ${ }^{3-7}$ To support

46 optimal growth and development, young children require nutrient dense diets so that nutrient

47 requirements may be achieved within appropriate levels of energy. ${ }^{8,9}$ The Dietary Guidelines for

48 Americans aged 2 years and older offer evidence-based recommendations for the prevention of

49 chronic disease over the lifespan, and have evolved from focus on individual dietary components

50 (e.g., nutrients and food groups) to focus on the dietary patterns, or combinations of foods

51 consumed. ${ }^{9}$ While evidence suggests most young children in the United States (US) consume

52 adequate amounts of many essential nutrients (e.g., protein, riboflavin), ${ }^{10,11}$ they also consume

53 inadequate amounts of vegetables, fruit, and whole grains ${ }^{12,13}$ and excessive amounts of sweet

54 and salty snacks and sugar-sweetened beverages. ${ }^{14-16}$ Overall, research indicates children in the

55 U.S. are not achieving dietary recommendations for healthy eating. ${ }^{17-19}$

56

57 Young children are dependent on their adult caregivers for the food and beverages provided. For

58 most children, the two most influential caregivers are their parents and early care and education

59 (ECE) providers. ${ }^{20}$ Hence, the home/family and ECE settings are critical environments for

60 shaping children's dietary intake. ${ }^{21,22}$ Given the importance of ECE, a federal food program, the

61 Child and Adult Care Food Program (CACFP), offers reimbursement to ECE programs that

62 serve nutritious meals and snacks to low-income children. ${ }^{23}$ There is evidence that participating

63 programs serve healthier foods and beverages and children consume healthier diets. ${ }^{24-26}$ While 
64 several studies have described young children's dietary intakes at home and/or at ECE

65 settings, ${ }^{27-39}$ there is little information, especially in the US, ${ }^{40,41}$ to account for or compare each

66 setting's contribution to total dietary intake. Furthermore, reports have often commented on

67 specific nutrients or food groups consumed and lack a more general interpretation of food

68 patterns or quality. Because the establishment of healthy dietary patterns during early childhood

69 is critical for lifelong health, ${ }^{1}$ a more nuanced and pragmatic understanding of the contributions

70 of home and ECE environments to the overall pattern and quality of children's consumption is

71 needed.

72

73 The aims of this study were to: 1) describe the total dietary intake among 3- to 4-year-old

74 children attending ECE centers in relation to age-appropriate recommendations from the Dietary

75 Guidelines for Americans and identify the proportion of consumption coming from ECE centers;

76 2) evaluate whether the quality of dietary intake patterns, as measured by the Healthy Eating

77 Index (HEI)-2015, ${ }^{42}$ at ECE centers is different from that when children are with parents; and 3)

78 assess whether the quality of dietary intake differs between weekdays (when children attend ECE

79 centers) and weekends. The authors hypothesized diet quality would be higher at ECE centers

80 than with parents and, subsequently, weekdays would exhibit higher diet quality than weekends.

81

\section{Materials and Methods}

\section{$83 \quad$ Participants and setting}

84 Data for this cross-sectional study were collected as part of a larger cluster randomized

85 controlled trial in central North Carolina evaluating the effectiveness of an 8-month social

86 marketing campaign (Healthy Me, Healthy We) to improve 3- to 4-year-old children's dietary 
87 and physical activity behaviors. ${ }^{43}$ Eligibility criteria for the larger trial specified that ECE centers

88 have at least one classroom with 3-4-year-old children, provide lunch, have a quality rating of 3-

895 stars (on a 5 -star scale) or be exempt from the quality rating, and not exclusively serve children

90 with special needs. Child participants had to be 3-4 years of age and enrolled at a participating

91 center. One parent participated with each child and provided written consent. The Institutional

92 Review Board at the University of North Carolina at Chapel Hill approved study protocols.

$94 \underline{\text { Measures }}$

95 Data collection occurred in two waves, prior to randomization, between July and September in

962015 and 2016. Data collection procedures included a combination of self-administered surveys,

97 physical assessments, and observation.

99 Demographics. Parents completed surveys containing questions about child age, sex, race, and 100 ethnicity, as well as parent sex, education, and household income. Center directors completed 101 surveys about the ECE center characteristics, including accreditation, subsidies, affiliations, and 102 total number of children enrolled.

104 Anthropometrics. Trained data collectors measured children's height and weight at their 105 respective ECE centers using standard protocols. ${ }^{43}$ Height and weight were used to calculate 106 body mass index (BMI, $\mathrm{kg} / \mathrm{m}^{2}$ ), and the Centers for Disease Control and Prevention growth 107 reference (2000) was used to determine age- and sex-specific BMI percentiles. ${ }^{44}$ Children with a 108 sex specific BMI-for-age at or above the $85^{\text {th }}$ percentile were classified as overweight or obese. 
109 Parents completed self-report measures for their height and weight; BMI was calculated using

110 these self-reported values.

111

112 Dietary Intake. To assess 24-hour intake across multiple settings and caregivers, dietary intake

113 was measured using a combination of observation and parent-report. ${ }^{45,46}$ Data were collected for

114 three days - two consecutive weekdays (Tuesday/Wednesday or Thursday/Friday) and one

115 weekend day. On weekdays, data collectors trained and certified on the Dietary Observation in

116 Child Care (DOCC) protocol observed and recorded all food and beverages consumed by

117 participating children while at the ECE center. ${ }^{47}$ Parents completed food records for

118 corresponding weekdays as well as one weekend day. Parents received instruction to document

119 all food and beverages consumed outside of child care in real-time, including brand names and

120 methods of preparation. A portion size estimation guide was provided and reviewed to aid

121 parents in estimating amounts children consumed. Members of the research team reviewed all

122 records - observation and parent-report - and contacted data collectors and parents, as needed,

123 within one week to clarify or collect additional information regarding the types or quantities of

124 food and drink consumed as well as potentially missing information (e.g., no drinks recorded).

126 Observed and parent-reported dietary intakes were merged to create 24-hour records that were 127 entered into the Nutrition Data System for Research (NDSR) software (versions 2015 and 128 2016). ${ }^{48}$ During entry, NDSR prompts location of meals and snacks, which provided the ability 129 to capture whether food was eaten at ECE centers (i.e., DOCC data) or any other location (i.e., 130 parent report). Final nutrient analyses were completed using NDSR version 2017 (July 2017) ${ }^{49}$ to 
131 obtain energy intake, macronutrients, micronutrients, and food group equivalents consistent with

132 units required to generate total and component HEI-2015 scores.

134 Dietary intake was summarized in regard to nutritional goals for the healthy US-style eating 135 pattern set forth by the 2015-2020 Dietary Guidelines for Americans for moderately active 3-4-

136 year-old boys and girls. ${ }^{9}$ Key elements include energy intake, macronutrient distribution (percent

137 energy intake from carbohydrates, fat, protein, as well as added sugar and saturated fat), trans fat,

138 sodium, and food group equivalents (fruit, vegetables, grains, protein, and dairy). Overall diet

139 quality was evaluated using the HEI-2015, a quantitative measure of alignment with the

140 Guidelines. ${ }^{42,50}$ The density-based scoring system (i.e., amount consumed per 1,000 kcal) allows

141 for the examination of quality of intake standardized for the quantity consumed. ${ }^{50,51}$ The HEI-

1422015 includes 13 components - nine adequacy components (dietary components to increase) and

143 four moderation components (dietary components to decrease). While most components are

144 standardized per calorie, the fatty acid (adequacy) component represents the ratio of healthier

145 unsaturated to less healthy saturated fats. Component scores typically range from 0 to 10 points,

146 but for components where one is a subset of another (e.g., Total Vegetables and Greens and

147 Beans), each component is scored 0 to 5 points. All components sum for a maximum score of

148 100, with a higher total HEI score indicating greater consistency with the Dietary Guidelines.

149 Summarizing dietary intake in these two manners provided the ability to view the absolute intake

150 of key nutrients and food groups, the nutrient density of foods and beverages consumed, as well

151 as an overall indicator of diet quality. 
153 Generating HEI scores. Distinct output files from the NDSR for total day and location-specific

154 (i.e., ECE center vs. all other locations) were imported to SAS version $9.4^{52}$ to calculate

155 component and total HEI-2015 scores for each child using publicly available SAS code from the

156 Nutrition Coordinating Center (University of Minnesota, Minneapolis). This simple HEI scoring

157 algorithm accounts for multiple days of intake per child by summing data across days prior to

158 generating a single set of standardized component and total scores for each child by setting and

159 weekday/weekend..$^{50}$

160

$161 \quad$ Statistical Analysis

162 This cross-sectional analysis comprised children for whom dietary intake was both observed at

163 the ECE center and reported for time outside of child care within a 24-hour period, including

164 children who provided only one of the intended two weekdays of record. Children with two

165 weekdays of intake were compared to children with only one weekday, but no differences were

166 detected between groups. Demographic characteristics of children and parents, characteristics of

167 centers, and children's dietary intakes and diet quality scores were summarized using descriptive

168 statistics, including frequencies for categorical data and means and standard deviations for

169 continuous data. Mixed effects models were used to determine whether mean HEI-2015

170 component and total scores differed by setting (i.e., ECE center or with parents) and weekdays

171 compared to weekend. These models included random intercepts to account for clustering within

172 ECE centers and children within families as well as accounting for repeated measures on

173 children. Children's age and sex were determined to be confounders a priori and were included

174 in adjusted models. In considering the opportunity for multiple comparisons within HEI-2015 
175 total and component scores, the alpha was adjusted using a Bonferroni correction and set at

$1760.00019(0.05 / 26$ comparisons $)$. All statistical analyses were performed in SAS version 9.4. ${ }^{52}$

\section{Results}

179 Sample Characteristics

180 Of the 908 children from 98 ECE centers who provided baseline data for the larger cluster

181 randomized controlled trial, 840 children met inclusion criteria for this analysis (Table 1).

182 Children with no dietary information were excluded $(n=2)$ as were those when weekday diet data

183 were missing corresponding pieces from the parent-reported records $(n=40)$ or observations at

184 the ECE center ( $n=26)$. Most children $(n=711)$ had two complete weekdays of record, while the

185 others $(\mathrm{n}=129)$ had one complete weekday of record. A majority of children had a day of record

186 for the weekend ( $\mathrm{n}=826)$, including children who only had information for one weekday $(93 \%)$.

188 A majority of children were white (45\%) or African American (38\%), and about one-quarter of

189 the children $(26 \%)$ were classified as overweight or obese (sex-specific BMI-for-age $\geq 85$ th

190 percentile). Parents completing study measures were primarily female (86\%) and a majority of

191 parents $(64 \%)$ were classified as overweight or obese $(\mathrm{BMI} \geq 25.0)$. Nearly half of the parents

192 (47\%) reported having a college or graduate-level degree and family income was reflective of

193 that in North Carolina. A variety of types of ECE centers were represented in the sample (e.g.,

194 faith-based, Head Start), and most accepted child care subsidies (92\%) and participated in the

$195 \operatorname{CACFP}(73 \%)$.

196

197 Dietary Intake 
198 Dietary Intakes. Children's dietary intakes at ECE centers and with parents, as well as on

199 weekdays and weekend days, are shown in Table 2. For parent-reported records, meals and

200 snacks were most frequently consumed at home (68\%) or 'other' locations that included

201 restaurants $(23 \%)$. Although specific dietary recommendations are dependent on the age and sex

202 of a child, the average 24-hour dietary intake for weekdays and weekends consistently fell short

203 of recommendations for vegetables, dairy, and whole grains and exceeded recommended limits

204 for sodium and percent energy intake from saturated fat and added sugars. On weekdays when

205 children consumed foods and beverages both at ECE and with parents, the majority of the total

206 day's energy (61\%), food groups (50-70\%), sodium (61\%), and added sugars (65\%) were

207 consumed with parents. When comparing weekdays and weekend days, children consumed more

208 energy and sodium, as well as a higher percent of energy from added sugar and saturated fat, on

209 weekends.

210

211 Quality of Intake by Setting. The mean total HEI-2015 scores for the food and beverage

212 consumed at ECE $(58.3 \pm 0.6)$ and with parents $(52.5 \pm 0.6)$ indicate children's dietary patterns

213 on the measured days of intake did not coincide with national recommendations at either setting

214 (Table 3). However, differences in scores generally suggest that children consumed a healthier

215 assortment of foods and beverages while at ECE. ${ }^{50}$ Statistically significant differences were

216 observed for 9 of the 13 HEI-2015 component scores, exceptions being greens and beans,

217 seafood and plant proteins, fatty acids, and sodium. Food and beverage consumed at ECE

218 appeared to provide more total fruit, whole fruit, whole grains, and dairy, as well as less added

219 sugars and saturated fats per calorie. Food consumed with parents provided more total vegetables

220 and total protein as well as fewer refined grains per calorie. Although many component scores 
221 across both settings were low, mean component scores less than 50\% of the maximum

222 component score help identify the greatest opportunities for improvement. Components with

223 these very low scores were similar across settings and included: total vegetables, greens and

224 beans, whole grains, seafood and plant proteins, and fatty acids.

225

226 Quality of Intake on Weekdays and Weekends. The mean total HEI-2015 scores for weekdays

$227(58.5 \pm 0.5)$ and weekends $(51.3 \pm 0.5)$ similarly showed that on the measured days of intake,

228 children did not consume diets consistent with recommendations for the prevention of chronic

229 disease (Table 3). The total HEI score for weekdays was higher than weekends. This difference

230 was statistically significant and may be clinically meaningful. ${ }^{50}$ Statistically significant

231 differences were observed for 8 of the 13 component scores, exceptions being total protein foods,

232 seafood and plant proteins, fatty acids, refined grains, and sodium. Weekday consumption

233 provided more total fruit, whole fruit, total vegetables, greens and beans, dairy, and whole grains,

234 as well as less added sugars and saturated fats per calorie. Regardless of the day of the week,

235 many component scores were low to moderate at best, and highlight opportunity for

236 improvement. The lowest component scores across weekday and weekend included total

237 vegetables, greens and beans, whole grains, seafood and plant proteins, fatty acids, refined

238 grains, and sodium.

239

240 Discussion

241 This cross-sectional analysis of observed dietary intake of 3- to 4-year-old children at ECE

242 centers and corresponding parent-reported intake outside of child care revealed, similar to results

243 from nationally representative samples of children who may or may not attend child care, that the 
244 quality of children's diets is low. ${ }^{12-19}$ Results also showed the quality of foods and beverages

245 children consumed at ECE centers was in fact higher than what was consumed elsewhere, as well

246 as on weekdays, when children are in child care, than on weekends, when they are not. Because

247 of the short-term and long-term effects of dietary intake during early childhood, improving diet

248 quality to ensure children meet nutrient recommendations within appropriate levels of energy

249 remains of critical importance. ${ }^{54}$

250

251 Previous investigations into the dietary intake of young children attending ECE centers have

252 either narrowly assessed setting (i.e., only ECE centers or only home), ${ }^{36-39}$ a portion of the day, ${ }^{41}$

253 or summarized total intake that was not identified by setting, ${ }^{28,32-35}$ making it difficult to draw

254 meaningful insights about the individual and complementary roles ECE centers and parents have

255 on children's dietary intake. Only one report (from 1999) had previously distinguished

256 consumption at ECE centers from home for an entire day. ${ }^{40}$ Results from this study mirror

257 previous findings in that children consumed inadequate amounts of food groups (e.g., vegetables

258 and whole grains) and other dietary constituents important for healthy development while also

259 consuming excess sodium and added sugars. ${ }^{28,32-35,40}$ However, differences in these results from

260 other studies signal that focusing on only portions of the day or absolute intake may misrepresent

261 total dietary intake. ${ }^{41}$

262

263 Based on the amount of time children spend in care outside the home, national guidelines suggest

264 that ECE provide up to one-half to two-thirds of children's daily nutrient requirements. ${ }^{55}$

265 However, results from this study, similar to findings estimating the proportion of preschool-aged

266 children's intake occurring outside of child care, ${ }^{36}$ indicate children consume approximately $40 \%$ 
267 of energy and nutrients at child care and instead consume about $60 \%$ of their diet with parents.

268 While this is less than current recommendations, the higher total HEI-2015 score at ECE centers

269 signifies children consumed more nutrient dense foods there than with parents. It is plausible that

270 participation in the CACFP provides children access to healthier foods and beverages within

271 ECE centers than what is provided by parents. ${ }^{33,38,39}$ In addition, lower total HEI-2015 scores

272 with parents may be related to the fact that approximately $12 \%$ of dietary intake of children aged

$2732-5$ years comes from quick-service restaurants. ${ }^{56}$ This not only affirms the critical role ECE

274 centers play in improving the overall quality of children's dietary intake, ${ }^{22}$ but also the

275 importance of synergy and partnership between ECE providers and parents to ensure children

276 have consistent access and encouragement to consume healthier foods and drinks..$^{21,40,57}$ Priorities

277 for improving quality of dietary intake are similar for ECE centers and families, and intervention

278 efforts should focus on improving the availability of healthy foods and encouraging children to

279 eat them. Specifically, the availability and consumption of more vegetables, particularly greens

280 and beans and seafood and plant proteins, less sodium and added sugars, and to substitute whole

281 grains for refined grains and polyunsaturated and monounsaturated fatty acids for saturated fats.

282

283 A key strength of the current study is the use of a quantitative measure of overall diet quality, the

284 HEI-2015 score. The density-based scoring system used to calculate the HEI-2015 separates diet

285 quality from quantity, which not only allows for interpretation of the nutrient density of the

286 combination of foods and beverages consumed but also for comparison across settings and days

287 in which absolute intake differed.$^{50}$ In addition, the component scores of the HEI-2015 make it

288 straightforward to identify specific targets for dietary interventions that can be translated to food-

289 based recommendations. For example, although children in this study, on average, consumed 
290 amounts of protein foods within range of recommendations, use of the HEI-2015 component

291 scores provided more detailed information that the quality of sources of protein could be

292 improved through substituting seafood and plant proteins.

294 Other assets of this study include the collection and data management of 24-hour dietary intake

295 in a large sample of children using a highly regarded diet analysis program (NDSR) in which

296 data across multiple settings and caregivers could be both distinguished and linked. While

297 dietary assessment in general is not without limitations, combining a valid and reliable direct

298 observation method ${ }^{47}$ with parent-reported food diaries minimized bias in obtaining 24-hour

299 intake for children who spend time with multiple caregivers. ${ }^{45,58}$ However, caution is warranted

300 when interpreting findings, as some differences may be the result of different methods of data

301 collection.

302

303 Other limitations include the generalizability of these results. This sample represents the types of

304 ECE centers and families receiving care in central North Carolina. However, results are

305 comparable to studies conducted in other states. ${ }^{28,32-35,40}$ Another limitation regarding

306 generalizability relates to the calculation of HEI-2015 scores with a simple scoring method. This

307 method is based on individuals' intake on the observed days; therefore it may not capture

308 episodic consumption of infrequently consumed food groups or subgroups, nor does it estimate

309 usual intake or adjust for measurement error. ${ }^{59}$ However, it can be used to estimate individual-

310 level scores that can then be used in more advanced statistical models. ${ }^{50,60}$ To answer these

311 research questions, it was necessary to use mixed effects models to control for clustering within

312 ECE centers, relationships between HEI-2015 scores and individual children's characteristics 
313 (i.e., age, sex), and the within subject comparison of looking at dietary intake by setting or

314 portion of the week (i.e., groups were not independent of one another).

\section{Conclusions}

317 This sample of 3- to 4-year-old children attending ECE centers in central North Carolina

318 consumed about $60 \%$ of their diet with parents and $40 \%$ at ECE centers. In general, children fell

319 short of recommendations for vegetables, dairy, and whole grains and exceeded

320 recommendations for sodium and percent energy intake from added sugars and saturated fats as

321 specified in the USDA Food Patterns. A deeper look at consumption specifically within ECE

322 centers and with parents revealed that children consumed higher quality foods and beverages at

323 ECE centers, most notably through more whole fruits, dairy, whole grains, and less added sugars

324 and saturated fat per calorie. Similarly, in comparing weekdays to weekends, children consumed

325 higher quality foods and beverages on weekdays than weekends. While ECE centers remain an

326 important source of nutrition for young children, there is still room for improvement. These

327 findings highlight the value of evaluating overall eating patterns, as opposed to specific nutrients

328 or portions of the day, and warrant further investigation about how to more effectively include

329 and support parents in fostering healthier eating environments so that children eat well across

330 settings and ultimately achieve higher quality dietary intake that positively influences longer-

331 term health. 


\section{References}

333 1. Institute of Medicine. From Neurons to Neighborhoods. Washington, D.C.: National 334 Academies Press; 2000.

335 2. Holt KA, Wooldridge NH, Story MT, Sofka D, eds. Bright Futures: Nutrition. $3^{\text {rd }}$ ed. $336 \quad$ Itasca, IL: The American Academy of Pediatrics; 2011.

337 3. Okubo H, Crozier SR, Harvey NC, et al. Diet quality across early childhood and adiposity at 6 years: the Southampton Women's Survey. Int J Obes. 2015;39(10):1456-1462.

339 4. Birch LL, Davison KK. Family environmental factors influencing the developing behavioral controls of food intake and childhood overweight. Pediatr Clin North Am. 2001;48(4):893-907.

342 5. Schwartz C, Scholtens PAMJ, Lalanne A, Weenen H, Nicklaus S. Development of healthy 343 eating habits early in life. Review of recent evidence and selected guidelines. Appetite. 2011;57(3):796-807.

6. Skinner JD, Carruth BR, Wendy B, Ziegler PJ. Children's food preferences: a longitudinal analysis. J Am Diet Assoc. 2002;102(11):1638-1647.

347 7. Gordon-Larsen P, The NS, Adair LS. Longitudinal trends in obesity in the United States from adolescence to the third decade of life. Obesity. 2010;18(9):1801-1804.

349 8. Brown JE, Isaacs JS, Krinke UB, et al. Nutrition Through the Life Cycle. 5th ed. Stamford, 350 CT: Cengage Learning; 2014.

3519 9.S. Department of Health and Human Services, U.S. Department of Agriculture. 2015 352 2020 Dietary Guidelines for Americans. 8th ed. US Dept of Health and Human Services website. https://health.gov/dietaryguidelines/2015/guidelines/. Published December 2015. $354 \quad$ Accessed September 16, 2019. 
355 10. Butte NF, Fox MK, Briefel RR, et al. Nutrient Intakes of US Infants, Toddlers, and 356 Preschoolers Meet or Exceed Dietary Reference Intakes. J Am Diet Assoc.

357 2010;110(12):S27-S37.

358 11. Bailey RL, Catellier DJ, Jun S, et al. Total Usual Nutrient Intakes of US Children (Under 35948 Months): Findings from the Feeding Infants and Toddlers Study (FITS) 2016. J Nutr. 360 2018;148(9S):1557S-1566S.

361 12. Guenther PM, Casavale KO, Kirkpatrick SI, et al. Diet Quality of Americans in 2001-02 362 and 2007-08 as Measured by the Healthy Eating Index-2010. Alexandria, VA: Center for 363 Nutrition Policy and Promotion, U.S. Department of Agriculture; 2013: Nutrition Insight 36451.

365 13. Welker EB, Jacquier EF, Catellier DJ, Anater AS, Story MT. Room for Improvement 366 Remains in Food Consumption Patterns of Young Children Aged 2-4 Years. J Nutr. 367 2018;148(9S):1536S-1546S.

368 14. Keast DR, Fulgoni VL, Nicklas TA, O’Neil CE. Food sources of energy and nutrients 369 among children in the United States: National Health and Nutrition Examination Survey 370 2003-2006. Nutrients. 2013;5(1):283-301.

371 15. Ford CN, Slining MM, Popkin BM. Trends in dietary intake among US 2- to 6-year-old 372 children, 1989-2008. J Acad Nutr Diet. 2013;113(1):35-42.

373 16. Kay M, Welker E, Jacquier E, et al. Beverage Consumption Patterns among Infants and 374 Young Children (0-47.9 Months): Data from the Feeding Infants and Toddlers Study, 375 2016. Nutrients. 2018;10(7):825.

376 17. Ogata BN, Hayes D. Position of the Academy of Nutrition and Dietetics: nutrition 377 guidance for healthy children ages 2 to 11 years. J Acad Nutr Diet. 2014;114(8):1257- 
1276.

379 18. Fox MK, Condon E, Briefel RR, Reidy KC, Deming DM. Food consumption patterns of

young preschoolers: are they starting off on the right path? J Am Diet Assoc. 2010;110(12 Suppl):S52-9.

382 19. Fox MK, Gearan E, Cannon J, et al. Usual food intakes of 2- and 3-year old U.S. children are not consistent with dietary guidelines. BMC Nutr. 2016;2(1):67.

20. Corcoran L, Steinley K, Grady S. Early Childhood Program Participation, Results from the National Household Education Surveys Program of 2016 (NCES 2017-101.REV). National Center for Education Statistics website. https://nces.ed.gov/pubs2017/2017101REV.pdf. Accessed September 16, 2019.

388 21. Skouteris H, McCabe M, Swinburn B, Newgreen V, Sacher P, Chadwick P. Parental 389 influence and obesity prevention in pre-schoolers: a systematic review of interventions. Obes Rev. 2011;12(5):315-328.

391 22. Larson N, Ward DS, Neelon SB, Story M. What Role Can Child-Care Settings Play in Obesity Prevention? A Review of the Evidence and Call for Research Efforts. J Am Diet Assoc. 2011;111(9):1343-1362.

394 23. U.S. Department of Agriculture, Food and Nutrition Service. Child and Adult Care Food 395 Program. US Dept of Agriculture website. https://www.fns.usda.gov/cacfp/child-andadult-care-food-program. Accessed September 16, 2019.

397 24. Ritchie LD, Boyle M, Chandran K, et al. Participation in the Child and Adult Care Food 398 Program Is Associated with More Nutritious Foods and Beverages in Child Care. Child Obes. 2012; 8(3):224-229.

400 25. Korenman S, Abner KS, Kaestner R, Gordon RA. The Child and Adult Care Food 
Program and the nutrition of preschoolers. Early Child Res Q. 2013;28(2):325-336.

402 26. Schwartz MB, Henderson KE, Grode G, et al. Comparing Current Practice to

403 Recommendations for the Child and Adult Care Food Program. Child Obes.

$404 \quad 2015 ; 11(5): 491-498$.

405 27. Goldbohm R, Rubingh C, Lanting C, Joosten K. Food Consumption and Nutrient Intake

406 by Children Aged 10 to 48 Months Attending Day Care in The Netherlands. Nutrients.

$407 \quad 2016 ; 8(7): 428$.

408 28. Larowe TL, Adams AK, Jobe JB, Cronin KA, Vannatter SM, Prince RJ. Dietary Intakes

409 and Physical Activity among Preschool Aged Children living in Rural American Indian

410 Communities Prior to a Family-based Healthy Lifestyle Intervention. J Am Diet Assoc.

$411 \quad 2010 ; 110(7): 1049-1057$.

412 29. Gubbels J, Raaijmakers L, Gerards S, Kremers S. Dietary Intake by Dutch 1- to 3-Year-

413 Old Children at Childcare and at Home. Nutrients. 2014;6(1):304-318.

414 30. Bernardi JR, Cezaro C De, Fisberg RM, Fisberg M, Vitolo MR. Estimation of energy and

415 macronutrient intake at home and in the kindergarten programs in preschool children. $J$

$416 \quad$ Pediatr. 86(1):59-64.

417 31. Gerritsen S, Anderson SE, Morton SM, Wall CR. Pre-school nutrition-related behaviours

418 at home and early childhood education services: findings from the Growing Up in New

419 Zealand longitudinal study. Public Health Nutr. 2018;21(07):1222-1231.

420 32. Bollella MC, Boccia LA, Nicklas TA, et al. Assessing dietary intake in preschool

421 children: The healthy start project - New York. Nutr Res. 1999;19(1):37-48.

422 33. Bruening KS, Gilbride JA, Passannante MR, McClowry S. Dietary Intake and Health

423 Outcomes among Young Children Attending 2 Urban Day-care Centers. J Am Diet Assoc. 
1999;99(12):1529-1535.

425 34. Bucholz EM, Desai MM, Rosenthal MS. Dietary intake in Head Start vs non-Head Start

426 preschool-aged children: results from the 1999-2004 National Health and Nutrition

427 Examination Survey. J Am Diet Assoc. 2011;111(7):1021-1030.

428 35. Mier N, Piziak V, Octelina Castillo-Ruiz, Velazquez G, Alfaro ME, Ramirez JA. Nutrition

429 Provided to Mexican-American Preschool Children on the Texas-Mexico Border. $J$ Am

$430 \quad$ Diet Assoc. 2007;107:311-315.

431 36. Robson SM, Khoury JC, Kalkwarf HJ, Copeland K. Dietary Intake of Children Attending 432 Full-Time Child Care: What Are They Eating Away from the Child-Care Center? J Acad $433 \quad$ Nutr Diet. 2015;115(9):1472-1478.

434 37. Padget A, Briley ME. Dietary Intakes at Child-Care Centers in Central Texas Fail to Meet 435 Food Guide Pyramid Recommendations. J Am Diet Assoc. 2005;105:790-793.

436 38. Romo-Palafox MJ, Ranjit N, Sweitzer SJ, et al. Adequacy of Parent-Packed Lunches and Preschooler's Consumption Compared to Dietary Reference Intake Recommendations. $J$ Am Coll Nutr. 2017;36(3):169-176.

439 39. Andreyeva T, Kenney EL, O’Connell M, Sun X, Henderson KE. Predictors of Nutrition Quality in Early Child Education Settings in Connecticut. J Nutr Educ Behav. 2018;50(5):458-467.

442 40. Briley ME, Jastrow S, Vickers J, Roberts-Gray C. Dietary intake at child-care centers and 443 away: Are parents and care providers working as partners or at cross-purposes? J Am Diet 444 Assoc. 1999;99:950-954.

445 41. Sisson SB, Kiger AC, Anundson KC, et al. Differences in preschool-age children's dietary 446 intake between meals consumed at childcare and at home. Prev Med reports. 2017;6:33- 
448 42. Krebs-Smith SM, Pannucci TE, Subar AF, et al. Update of the Healthy Eating Index: HEI2015. J Acad Nutr Diet. 2018;118(9):1591-1602.

450 43. Hennink-Kaminski H, Vaughn AE, Hales D, Moore RH, Luecking CT, Ward DS. Parent and child care provider partnerships: Protocol for the Healthy Me, Healthy We (HMHW) cluster randomized control trial. Contemp Clin Trials. 2018;64:49-57.

453 44. Kuczmarski RJ, Ogden CL, Guo SS, et al. 2000 CDC Growth Charts for the United States: methods and development. Vital Health Stat 11. 2002;(246):1-190.

455 45. Baranowski T, Sprague D, Baranowski JH, Harrison JA. Accuracy of maternal dietary recall for preschool children. J Am Diet Assoc. 1991;91(6):669-674.

457 46. Burrows TL, Martin RJ, Collins CE. A Systematic Review of the Validity of Dietary Assessment Methods in Children when Compared with the Method of Doubly Labeled Water. J Am Diet Assoc. 2010;110(10):1501-1510.

47. Ball SC, Benjamin SE, Ward DS. Development and Reliability of an Observation Method to Assess Food Intake of Young Children in Child Care. J Am Diet Assoc. 2007;107(4):656-661.

463 48. NDSR [computer program]. Version 2016. Minneapolis, MN: University of Minnesota $464 \quad$ Nutrition Coordinating Center; 2016.

465 49. NDSR [computer program]. Version 2017. Minneapolis, MN: University of Minnesota $466 \quad$ Nutrition Coordinating Center; 2017.

467 50. Kirkpatrick SI, Reedy J, Krebs-Smith SM, et al. Applications of the Healthy Eating Index 468 for Surveillance, Epidemiology, and Intervention Research: Considerations and Caveats. $J$ 469 Acad Nutr Diet. 2018;118(9):1603-1621. 
470 51. Guenther PM, Casavale KO, Reedy J, et al. Update of the Healthy Eating Index: HEI471 2010. J Acad Nutr Diet. 2013;113(4):569-580.

472 52. SAS [computer program]. Version 9.4. Cary, NC: SAS Institute, Inc.; 2018.

473 53. Stallings VA, Harrison M, Oria M, eds. Dietary Reference Intakes for Sodium and $474 \quad$ Potassium. Washington, D.C.: National Academies Press; 2019.

475 54. Dietary Guidelines Advisory Committee. Scientific Report of the 2015 Dietary Guidelines 476 Advisory Committee: Advisory Report to the Secretary of Health and Human Services and the Secretary of Agriculture. US Dept of Health and Human Services website. https://health.gov/dietaryguidelines/2015-scientific-report/PDFs/Scientific-Report-of-the2015-Dietary-Guidelines-Advisory-Committee.pdf. Accessed September 16, 2019.

480 55. Benjamin-Neelon SE. Position of the Academy of Nutrition and Dietetics: Benchmarks 481 for Nutrition in Child Care. J Acad Nutr Diet. 2018;118(7):1291-1300.

482 56. U.S. Department of Agriculture, Agricultural Research Service. Food Patterns Equivalents 483 Intakes from Food: Mean Amounts Consumed per Individual, by Family Income as \% of Poverty Level and Age, What We Eat in America, NHANES 2015-2016. US Dept of Agriculture website. www.ars.usda.gov/nea/bhnrc/fsrg. Accessed September 16, 2019.

486 57. Ward DS, Welker E, Choate A, et al. Strength of obesity prevention interventions in early $487 \quad$ care and education settings: A systematic review. Prev Med (Baltim). 2017;95:S37-S52.

488 58. Thompson FE, Kirkpatrick SI, Subar AF, et al. The National Cancer Institute's Dietary 489 Assessment Primer: A Resource for Diet Research. J Acad Nutr Diet. 2015;115(12):1986490 1995.

491 59. Epidemology and Genomics Research Program. Overview of the Methods \& Calculations. 492 National Cancer Institute website. https://epi.grants.cancer.gov/hei/hei-methods-and- 
$493 \quad$ calculations.html. Accessed September 16, 2019.

494 60. Thomson JL, Tussing-Humphreys LM, Landry AS, Goodman MH. No Improvements in 495 Postnatal Dietary Outcomes Were Observed in a Two-Arm, Randomized, Controlled, 496 Comparative Impact Trial among Rural, Southern, African-American Women. J Acad $497 \quad$ Nutr Diet. 2018;118(7):1196-1207.

498 
Table 1. Demographic and anthropometric characteristics of 840 parent-child dyads from 98 early care and education centers in North Carolina in 2015 - 2016 who had at least one weekday record containing dietary intake at both child care and with parents and characteristics of centers

\section{Children}

mean $\pm S D^{a}$

Age, years $(n=837)$

$$
4 \pm 0.6
$$

$$
n(\%)
$$

Sex, female $(\mathrm{n}=838)$

Race $(n=797)$

White

Black or African American

305 (38.2)

Asian

American Indian or Alaska Native

More than one race

Not specified

Ethnicity $(\mathrm{n}=808)$

Hispanic or Latino

Anthropometric $(\mathrm{n}=836)$ mean $\pm S D$

$\mathrm{BMI}^{\mathrm{b}}$ percentile $61.3 \pm 27.8$ $n(\%)$ 
BMI-for-age $\geq 85$ th percentile

$214(25.6)$

\section{Parents}

$n(\%)$

Sex, female $(n=819)$

$704(86.0)$

Highest level of education completed $(n=804)$

High school diploma/GED or lower

$134(16.7)$

Some college

Associate degree

$101(12.6)$

College degree or higher

$378(47.0)$

Annual family household income $(\mathrm{n}=717)$

Under $\$ 30,000$

$242(33.8)$

$\$ 30,000-\$ 59,999$

$158(22.0)$

$\$ 60,000$ or more

$317(44.2)$

mean $\pm S D$

BMI $(n=779)$

$28.8 \pm 7.4$

Weight status

$n(\%)$

Underweight $(\mathrm{BMI}<18.5)$

$11(1.4)$

Normal weight (BMI 18.5 to $<25$ )

$269(34.5)$

Overweight (BMI 25.0 to $<30$ )

$230(29.5)$

Obese (BMI $\geq 30.0)$

$269(34.5)$

Centers

n (\%)

Accredited by the National Association for the

Education of Young Children

$22(22.4)$ 
Participates in the Child and Adult Care Food

Program

Other program affiliations ${ }^{\mathrm{c}}$

NC Pre-K or other pre-kindergarten

Faith-based

Head Start and/or Early Head Start

Military

Native American or Alaska Native tribe
Accepts child care subsidies

$90(91.8)$

$71(72.5)$

$33(33.7)$

$20(20.4)$

$11(11.2)$

$3(3.1)$

$1(1.0)$

mean, range

Total child enrollment

$87,28-218$

\footnotetext{
${ }^{\mathrm{a}} \mathrm{SD}=$ standard deviation

${ }^{\mathrm{b}} \mathrm{BMI}=$ body mass index, calculated as $\mathrm{kg} / \mathrm{m}^{2}$

${ }^{\mathrm{c}}$ Centers could select all options that applied
} 
Table 2. Dietary intakes of 3-4-year-old children attending center-based early care and education in central North Carolina in 2015 2016

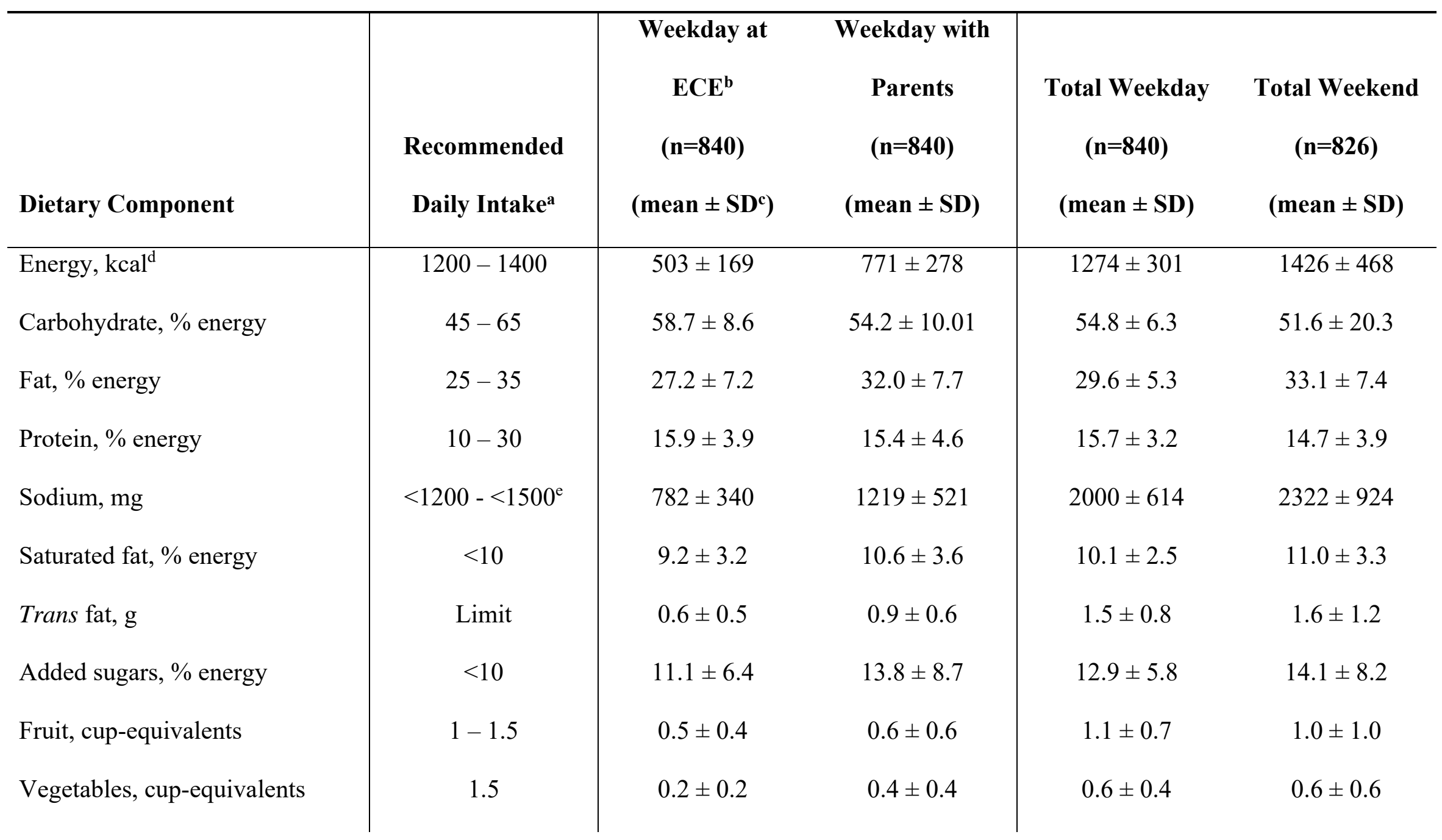




\begin{tabular}{l|c|c|cc} 
Grains, ounce-equivalents & $4-5$ & $2.1 \pm 0.9$ & $2.7 \pm 1.5$ & $4.8 \pm 1.7$ \\
Whole grains, ounce-equivalents & $2-2.5$ & $0.4 \pm 0.5$ & $0.4 \pm 0.6$ & $0.8 \pm 0.8$ \\
Protein foods, ounce-equivalents & $3-4$ & $0.9 \pm 0.8$ & $2.0 \pm 1.4$ & $2.9 \pm 1.7$ \\
Dairy, cup-equivalents & 2.5 & $1.1 \pm 0.6$ & $1.0 \pm 0.8$ & $2.1 \pm 1.0$ \\
\end{tabular}

${ }^{\mathrm{a}}$ Based on Dietary Reference Intakes and Healthy US-style USDA Food Patterns for moderately active 3-4-year-old girls and boys

${ }^{\mathrm{b}} \mathrm{ECE}=$ early care and education

${ }^{\mathrm{c}} \mathrm{SD}=$ standard deviation

${ }^{\mathrm{d}}$ Energy is based on estimated average requirements, not a recommended daily intake

${ }^{\text {e }}$ Chronic Disease Risk Reduction Intake ${ }^{53}$ 
Table 3. Healthy Eating Index-2015 scores ${ }^{\mathrm{a}}$ by setting and day of the week of 3-4-year-old children attending center-based early care and education in central North Carolina in 2015 - 2016

\begin{tabular}{|c|c|c|c|c|c|c|c|}
\hline HEI-2015 ${ }^{\text {b }}$ Component $^{-}$ & $\begin{array}{c}\text { Maximum } \\
\text { Points }\end{array}$ & $\begin{array}{c}\text { Weekday at } \\
\text { ECE }^{c, d} \\
(n=840) \\
\left(\text { mean } \pm S^{e}\right)\end{array}$ & $\begin{array}{c}\text { Weekday } \\
\text { with } \\
\text { Parents }^{d} \\
(n=840) \\
\text { (mean } \pm \text { SE) }\end{array}$ & p-value ${ }^{f}$ & $\begin{array}{c}\text { Total } \\
\text { Weekday } \\
(\mathbf{n}=840) \\
(\text { mean } \pm \text { SE) }\end{array}$ & $\begin{array}{l}\text { Total Weekend } \\
\qquad(n=826) \\
(\text { mean } \pm \text { SE })\end{array}$ & p-value \\
\hline \multicolumn{8}{|l|}{ Adequacy } \\
\hline Total fruits & 5 & $4.2 \pm 0.1$ & $3.0 \pm 0.1$ & $<0.0001$ & $4.0 \pm 0.1$ & $3.1 \pm 0.1$ & $<0.0001$ \\
\hline Whole fruits & 5 & $4.3 \pm 0.1$ & $2.8 \pm 0.1$ & $<0.0001$ & $4.1 \pm 0.1$ & $2.9 \pm 0.1$ & $<0.0001$ \\
\hline Total vegetables & 5 & $1.8 \pm 0.1$ & $2.3 \pm 0.1$ & $<0.0001$ & $2.2 \pm 0.1$ & $1.9 \pm 0.1$ & $<0.0001$ \\
\hline Greens and beans & 5 & $0.9 \pm 0.1$ & $1.2 \pm 0.1$ & 0.001 & $1.4 \pm 0.1$ & $0.9 \pm 0.1$ & $<0.0001$ \\
\hline Whole grains & 10 & $4.3 \pm 0.2$ & $3.1 \pm 0.2$ & $<0.0001$ & $4.1 \pm 0.2$ & $3.1 \pm 0.2$ & $<0.0001$ \\
\hline Dairy & 10 & $9.3 \pm 0.1$ & $7.2 \pm 0.1$ & $<0.0001$ & $9.0 \pm 0.1$ & $7.0 \pm 0.1$ & $<0.0001$ \\
\hline Total protein foods & 5 & $2.9 \pm 0.1$ & $3.8 \pm 0.1$ & $<0.0001$ & $3.8 \pm 0.1$ & $4.0 \pm 0.1$ & 0.004 \\
\hline Seafood and plant proteins & 5 & $1.2 \pm 0.1$ & $1.4 \pm 0.1$ & 0.159 & $1.9 \pm 0.1$ & $1.6 \pm 0.1$ & 0.01 \\
\hline
\end{tabular}




\begin{tabular}{|c|c|c|c|c|c|c|c|}
\hline Fatty acids & 10 & $4.6 \pm 0.2$ & $4.8 \pm 0.2$ & 0.245 & $4.5 \pm 0.2$ & $5.1 \pm 0.2$ & 0.0003 \\
\hline \multicolumn{8}{|l|}{ Moderation } \\
\hline Refined grains & 10 & $4.4 \pm 0.2$ & $5.5 \pm 0.2$ & $<0.0001$ & $4.9 \pm 0.1$ & $4.9 \pm 0.1$ & 0.63 \\
\hline Sodium & 10 & $5.3 \pm 0.2$ & $4.9 \pm 0.2$ & 0.009 & $4.9 \pm 0.1$ & $4.5 \pm 0.1$ & 0.002 \\
\hline Added sugars & 10 & $7.4 \pm 0.1$ & $6.2 \pm 0.1$ & $<0.0001$ & $6.7 \pm 0.1$ & $6.1 \pm 0.1$ & $<0.0001$ \\
\hline Saturated fats & 10 & $7.7 \pm 0.1$ & $6.5 \pm 0.1$ & $<0.0001$ & $7.1 \pm 0.1$ & $6.1 \pm 0.1$ & $<0.0001$ \\
\hline Total HEI-2015 Score & 100 & $58.3 \pm 0.6$ & $52.5 \pm 0.6$ & $<0.0001$ & $58.5 \pm 0.5$ & $51.3 \pm 0.5$ & $<0.0001$ \\
\hline
\end{tabular}

${ }^{\text {a }}$ Scores adjusted for child sex, age in years, and account for clustering by centers and repeated measures on children

${ }^{\mathrm{b}}$ HEI-2015 = Healthy Eating Index-2015

${ }^{\mathrm{c}} \mathrm{ECE}=$ early care and education

${ }^{\mathrm{d}}$ Based only on weekday records of intake

${ }^{\mathrm{e}} \mathrm{SE}=$ standard error

${ }^{\mathrm{f}} \mathrm{p}$-values bolded denote those that reached statistical significance for correcting for multiple comparisons using Bonferroni correction ( $\mathrm{p}<0.00019$ ) 